RMD Open

Rheumatic \&

Musculoskeletal Diseases

\title{
A prospective study of the 1-year incidence of fibromyalgia after acute whiplash injury
}

\section{Robert Ferrari}

To cite: Ferrari R. A prospective study of the 1 year incidence of fibromyalgia after acute whiplash injury. RMD Open 2015;1:e000007. doi:10.1136/rmdopen-2014000007

- Prepublication history for this paper is available online. To view these files please visit the journal online (http://dx.doi.org/10.1136/ rmdopen-2014-000007).

Received 20 October 2014 Revised 28 November 2014 Accepted 1 December 2014

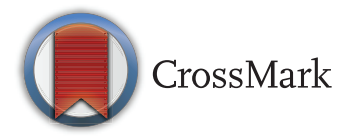

Department of Medicine, University of Alberta, Edmonton, Alberta, Canada

Correspondence to Dr Robert Ferrari; rferrari@shaw.ca

\section{ABSTRACT}

Objective: To measure the 1-year incidence of fibromyalgia in a cohort of acute whiplash-injured participants.

Methods: Consecutive acute patients with whiplash were assessed via the 2010 Modified American College of Rheumatology (ACR) criteria for fibromyalgia at 3 months, 6 months and 1 year postinjury. At each of these follow-up points, participants were also examined for recovery from whiplash injury.

Results: Of an initial 268 participants, data on recovery was available for 264 participants during the 1-year follow-up period. At the 3-month follow-up, $62 \%(167 / 268)$ of participants reported recovery from their whiplash injuries. At 6 months, $76 \%$ (203/268) reported recovery, and at 1 year $82 \%(216 / 264)$ reported recovery. At 3 and 6 months follow-up none of the participants met the 2010 Modified ACR Criteria for fibromyalgia, but fibromyalgia criteria were met for 2 (of 264) seen at the 1-year follow-up, yielding a 1-year incidence of $0.8 \%(95 \% \mathrm{Cl} 0.1 \%$ to $3.0 \%)$.

Conclusions: In the primary care setting, a significant proportion of patients with whiplash recover from whiplash injury at 1 year, and the incidence of fibromyalgia after acute whiplash injury is very low. The impression that fibromyalgia is common after whiplash injury may be due to the failure to exclude precollision fibromyalgia cases or due to referral bias of non-recovered patients.

It has been demonstrated, in the primary care setting, that a significant proportion of patients will recover from whiplash injury by 3 months postinjury, ${ }^{1-5}$ and even at 1 year postinjury the recovery rate can approach $80 \%{ }^{6}$ This still leaves $20 \%$ who fail to recover, and who are at risk not only for chronic pain, but according to some, for fibromyalgia. ${ }^{7}$ There are a number of studies or reports that have considered the incidence of fibromyalgia after whiplash or other physical injury, and the matter remains controversial. ${ }^{8}$ For the most part, studies have suffered from severe selection bias ${ }^{9}$ and failure to account for pretrauma fibromyalgia. ${ }^{10}$ Besides selection bias, misclassification is of

\section{Key messages}

The incidence of fibromyalgia after whiplash injury is a controversial topic.

- This study excludes pre-existing fibromyalgia to avoid selection bias encountered in previous studies.

- In the current study, the one year incidence of fibromyalgia after whiplash injury is on the order of $0.8 \%$.

- This study provides an upper limit for the incidence of fibromyalgia after whiplash injury, but does not deal with causation.

particular concern, where patients with acute whiplash who have precollision widespread pain, with or without a diagnosis of fibromyalgia, are included in the study. In addition, there is the problem that the denominator for calculation of incidence of fibromyalgia after trauma may be underestimated. That is, many patients with whiplash often do well, recovering quickly, and are thus not included in the prospective or retrospective determination of incidence because they do not come to medical attention. Thus, the denominator for considering the incidence of fibromyalgia is much reduced, falsely elevating the incidence.

Even in the primary care setting, there is selection bias, as some patients with whiplash can be injured yet choose never to see a physician or any therapist, but fully recover. ${ }^{11}$ Nevertheless, the primary care setting is a better opportunity to reduce selection bias and provide a more accurate estimation of the incidence of fibromyalgia following acute whiplash injury than are secondary treatment centre population samples or nonconsecutively collected participants. The purpose of the current study was to prospectively evaluate acute whiplash-injured participants in the primary care setting and determine the incidence of fibromyalgia at 1-year postinjury. 


\section{METHODS}

\section{Participants}

The author recruited a cohort of consecutive whiplashinjured patients presenting within 14 days of their collision to one of four primary care centres. Patients with a motor vehicle collision and suspected whiplash-associated disorder (WAD) were routinely referred from general practitioners at the clinic, directly to the author, who was acting as a specialist consultant within that clinic. The specialist was an internist with an interest in rheumatology and chronic pain. It was the practice during the time of this consultant's presence at the clinics to refer all acute patients with whiplash to the consultant for comanagement.

All participants were, at the time of the study, in a system of new legislation that places a cap on compensation for whiplash grade 1 and 2, of $\$ 4000$, with a standardised diagnostic treatment protocol applied to each participant. This system has been described elsewhere. ${ }^{12}$ All participants had filed a claim with an insurance company to receive treatment benefits. Twelve participants had experienced their collisions in the course of work, and thus were covered (in terms of treatment) by the provincial Workers' Compensation Board.

\section{Inclusion and exclusion criteria}

Prospective participants were further assessed for inclusion and exclusion criteria at the time of initial interview. Participants were examined to determine their WAD grade. ${ }^{13}$ WAD grade 1 or 2 patients were included if they were seated within the interior of a car, truck, sports/utility vehicle or van in a collision (any of rear, frontal or side impact), had no loss of consciousness, were 18 years of age or above, and presented within 14 days of their collision. Patients were excluded if they were told they had a fracture or neurological injury (ie, grade 3 or grade $4 \mathrm{WAD}$ ), had objective neurological signs on examination (loss of reflexes, sensory loss-ie, grade $3 \mathrm{WAD}$ ), no fixed address or current contact information, were unable to communicate in English, had acute non-traumatic neck pain or were admitted to hospital. In addition, participants who had additional collisions after the initial assessment were excluded so as not to have to restart the timing of the 1-year follow-up. As part of the objective was a determination of the incidence of fibromyalgia, participants who had a diagnosis of fibromyalgia (either by rheumatologist diagnosis, the 1990 American College of Rheumatology (ACR) criteria $^{14}$ or the Modified 2010 ACR (ACR) criteria ${ }^{15}$ ) or confirmed widespread pain ${ }^{15}$ prior to the collision were also excluded. Furthermore, pre-existing chronic pain conditions (such as rheumatoid arthritis, chronic mechanical spinal pain, osteoarthritis, or even previous WAD without widespread pain or secondary fibromyalgia), were not a reason for exclusion.

\section{Data collection and measurements}

The author gathered data on these participants referred over a 9-month period in 2012-2013, the measurements being conducted at the initial and follow-up consultations as part of the routine measures provided to all patients (ie, as part of usual assessment).

\section{Recovery from whiplash injury}

At 3 months, 6 months, and 1 year postinjury, recovery was assessed with the question: "Do you feel you have recovered from your injuries?" with responses of "yes', 'no', or 'not sure'. This has been shown to be as useful as completing a whiplash disability questionnaire and other similar measures of recovery. ${ }^{16}$

\section{Diagnosis of fibromyalgia}

The diagnosis of fibromyalgia was established by the Modified 2010 (ACR) criteria questionnaire. ${ }^{15}$ The Modified 2010 ACR criteria for fibromyalgia indicate that a diagnosis of fibromyalgia is satisfied when the following three conditions are met: (1) the Widespread Pain Index is $\geq 7$ and the Symptom Severity Score is $\geq 5$, or the Widespread Pain Index is 3-6 and the Symptom Severity Score is $\geq 9$; (2) symptoms have been present at a similar level for at least 3 months; and, (3) the patient does not have a disorder that would otherwise explain the pain. Recent studies have shown that these criteria can be examined by a questionnaire that can be completed by patients without a clinical examination, and that a total score of $>12$ (of a maximum of 31) has a high sensitivity and specificity for the gold standard diagnosis of fibromyalgia by a rheumatologist. ${ }^{17}{ }^{18}$ In order to not bias the author or other treating physicians, the participants completed the Modified 2010 ACR criteria questionnaire on their own, then the patient placed the questionnaire results in an envelope, numbered and matched to their name at 3 months, 6 months, and 1 year postinjury. This data were kept from the author's knowledge until 1 year follow-up was complete. Then, after an anonymisation process (each patient's name removed and replaced with their envelope number), data entry took place.

Participants were asked to return for 3-month, 6-month and 1-year assessments even if improved or recovered. Since the author was also involved in the care of these participants for other medical problems, the author also used these opportunities to capture data on recovery and obtain completed the Modified 2010 ACR criteria questionnaires at or near the follow-up periods, as did the involved primary care physicians and associated nursing staff. Where necessary, the questionnaires were mailed to patients or they were contacted to come to the clinic to complete them, and multiple efforts were made to obtain the highest rate of questionnaire return. At the same time, staff and other physicians assisted in ensuring the patients completed and returned their questionnaires as near to the follow-up times as possible. The Modified 2010 ACR criteria questionnaire was not labelled as a 'fibromyalgia' questionnaire, but rather a 'follow-up general health questionnaire'. 


\section{Data analysis}

At approximately 1 year postinjury for each participant, after final questionnaires were obtained, all data records were reviewed in order to ascertain if any data issues, such as missing data, outliers or out of range values, existed within the data set. Rather than exclude the participant, if there was no recorded number for any of the three symptoms of abdominal pain, fatigue or depression, then that participant was given the maximum score of three for those items. This biased the study towards an increased likelihood of diagnosing fibromyalgia and allowed the retention of these participants in the study, acting, in effect, as a sensitivity measure. The mean age, sex distribution and mean Modified 2010 ACR criteria scores were calculated at the 3-month, 6-month, and 1-year follow-up. The proportion of participants who recovered was also calculated at each follow-up, as well as the proportion of participants who met the case definition of fibromyalgia by the Modified 2010 ACR criteria questionnaire score of $>12$. The incidence of fibromyalgia at 1 year was calculated by dividing the proportion of participants meeting the fibromyalgia diagnosis criteria by the total number of participants for which data were available at that follow-up. Any participants diagnosed with fibromyalgia were then examined to assess if there was any other explanation for the widespread pain.

\section{Sample size calculation}

Previous prospective studies of the incidence of fibromyalgia after whiplash injury have suggested an incidence from $1 \%$ to $3 \% .^{8}$ Using this range, the author calculated that at least 250 participants would be required to provide an $80 \%$ power to detect a proportion with a $95 \%$ upper CI of $3 \%$. The author thus aimed for a sample size of at least 250 participants total.

\section{Ethics}

Ethical clearance was obtained from the Alberta Health Ethics Research Board.

\section{RESULTS}

\section{Data cleaning}

Initially, a total of 324 participants were evaluated. From these, 56 were eventually excluded (20 due to history of fibromyalgia or widespread pain preceding the collision, 12 due to lack of ability to communicate in English, 10 due to a new collision or injury event in the period of the study, 4 due to having been admitted to hospital, 7 due to loss of consciousness, 2 due to grade $3 \mathrm{WAD}$, and 1 due to grade 4 WAD). Thus, 268 participants formed the cohort for the study.

The questionnaires were completed over a range of time periods, from 12 to 16 weeks at the initial follow-up, 22-27 weeks for the second follow-up and 12-14 months for the final follow-up. It was learned that at least 44 participants had lost or misplaced their questionnaires and had returned at various points to obtain another one from staff. This largely explains the varying periods of follow-up.

At the designated 3 months follow-up, one participant was at first lost to follow-up, but when subsequently and coincidentally seen at 8 months postinjury, had reported recovery that he deemed to have taken place in a few weeks postinjury. Thus, this participant's data were included. At 6 months follow-up, an additional seven participants were lost to follow-up. All of these had reported recovery at 3 months, however, and when seen more than a year postcollision for other reasons, both their reports and chart notes as a well as medicolegal reports confirmed recovery within 6 months. Thus, their recovery data were included at 6 months and 1-year as recovered. At 1 year follow-up, 12 participants were lost to follow-up, but 8 of these had recorded recovery at 6 months, and indeed none of these had fibromyalgia at 6 months. Thus, there were only four participants lost to follow-up whose recovery status at 1 year and Modified 2010 ACR Modified criteria questionnaire scores at 1 year were unknown. In summary, the losses to follow-up for the outcomes of interest were 4 of 268 at 1 year postinjury.

\section{Descriptive statistics}

The mean age of the cohort was $38.5( \pm 12.5)$ years with $54 \%$ females.

\section{Recovery from whiplash injury}

Of an initial 268 participants, 264 participants were designated as followed up at 1 year. At the 3-month follow-up, 62\% (167/268) of participants reported recovery from their injuries. At 6 months, $76 \%$ (203/268) reported recovery, and at 1 year $82 \% \quad(216 / 264)$ reported recovery.

\section{Modified 2010 ACR criteria questionnaire scores}

At 3 months follow-up, none of the participants met the Modified 2010 ACR criteria for fibromyalgia, but at 6 months follow-up, three participants had scores that met the Modified 2010 ACR criteria for fibromyalgia. When further assessed, one of these patients was found to have polymyalgia rheumatica and subsequently responded to treatment, another was diagnosed with ankylosing spondylitis, responding eventually to nonsteroidal anti-inflammatory drugs, and another was found to have polymyositis, also responding to treatment. Thus, there were no fibromyalgia cases at 6 months follow-up. Fibromyalgia criteria were met, however, for 2 (of 264) at the 1-year follow-up, with no alternative explanation for symptoms. This yields a 1-year incidence of $0.8 \%$ (95\% CIs of $0.1 \%$ to $3.0 \%$ ). There was one male and one female fibromyalgia participant. If one includes the four participants lost to follow-up at 1 year who had still not recovered after 6 months as potentially having developed fibromyalgia, then the incidence could be $2.2 \%$ (6/268), with $95 \%$ 
CIs of $1.0 \%$ to $5.0 \%$. It should be noted that it was possible, through Netcare (the province-wide electronic healthcare database used in Alberta, Canada) review to examine those four participants lost to follow-up. Of those four participants lost to follow-up, only one had healthcare record evidence (eg, based on available specialist reports and medications), of an ongoing chronic pain disorder at 18 months postinjury. Thus, it is highly unlikely that all four had fibromyalgia.

Six of the participants went from recovered to nonrecovered status over the period of this study, but all these had recovered at 1 year follow-up.

\section{DISCUSSION}

This prospective study of the incidence of fibromyalgia after acute whiplash injury shows that the incidence is low, on the order of $0.8 \%$. This is in keeping with the observations of others. ${ }^{8} 10$ This study helps to define an 'upper limit' for the incidence of fibromyalgia after acute whiplash injury, but does not confirm causation. That is, the two participants who developed fibromyalgia may or may not have developed this condition because they suffered acute whiplash injury. Causation is a complex medical and legal topic. ${ }^{8}$ Croft et al, ${ }^{19}{ }^{20}$ for example, have shown that the prevalence of widespread pain in the general population is as much as $11 \%$, and that $20 \%$ of those identified as having chronic widespread pain likely meet diagnostic criteria for fibromyalgia. ${ }^{20}$ This is a prevalence of at least $2.2 \%$. Thus, acute whiplash injury may simply have been coincident with the development of fibromyalgia in the two participants in the current study. Causation between acute whiplash injury and fibromyalgia should not be implied via the results of the current study. Indeed, as has long been surmised, "It is incident to physicians, I am afraid, beyond all other men, to mistake subsequence for consequence". ${ }^{21}$

One strength of this study is the high rate of follow-up, even if not at all the designated time points, to confirm recovery and obtain completed Modified 2010 ACR criteria questionnaires. The key strength of this study, however, is the exclusion of patients with pre-existing widespread pain and fibromyalgia. This may indeed be the main flaw of previous studies. ${ }^{8}$ Without extensive examination of the patient's history directly from the patient, through pre-existing charts and/or electronic health records, it is possible that some studies include pre-existing cases of fibromyalgia in their study cohorts purporting to assess new cases of fibromyalgia after acute whiplash injury. The current study avoids this, but clearly, had the 20 participants with pre-existing fibromyalgia not been excluded, the results of this study would have been quite different. This explains perhaps the anecdotal observations of physicians that fibromyalgia is common after a collision. It is, in fact, common enough before a collision that it must be excluded in a study considering the incidence of new cases. It is of interest, furthermore, that four of those participants with a precollision diagnosis of fibromyalgia denied any significant pain precollision. Yet, they had an extensive history of chronic pain on chart review and had been seen by and diagnosed by a rheumatologist as having fibromyalgia. Another strength of the study is that it was biased towards including participants with other painful conditions precollision and assuming participants with missing data would endorse symptoms. A final strength is that the diagnosis of fibromyalgia was not based on clinical examination of, for example, tender points, where physician bias could have affected the diagnosis incidence.

The results are not surprising, as $80 \%$ of the acute whiplash-injured participants had recovered at 1 year. This leaves only $20 \%$ who could be at risk for fibromyalgia. When one examines the non-recovered participants, however, even though they have chronic pain and disability, they often report very localised pain disorders, such as chronic headache, chronic neck pain only or even chronic low back pain only. In fact, in another study, ${ }^{17}$ Ferrari and Russell examined 277 participants with widespread pain, mainly with labels of WAD, osteoarthritis, tendinitis, bursitis and mechanical spinal pain. Less than $10 \%$ of these met the Modified 2010 ACR criteria for fibromyalgia. What distinguished fibromyalgia patients from, for example, patients with WAD in that study was not chronic or widespread pain, but rather the endorsement of severe depression, severe fatigue, severe abdominal pain and multiple sites of pain (often eight or more). Patients with whiplash, even when nonrecovered, tend to have few sites of pain. In the current study, one case of non-recovered whiplash participant at 1 year, for example, reported only migraine headaches and another only left shoulder pain (later found to have a labrum tear). Thus, even among non-recovered participants, there are either few cases of widespread pain, or despite widespread pain, there is little in the way of depression, fatigue or abdominal pain. Thus, few are at risk for fibromyalgia. The failure to exclude pre-existing fibromyalgia and the selection bias that occurs in some studies may explain the previously held belief that fibromyalgia after whiplash injury is common.

One limitation of the study includes the fact that the gold standard diagnosis (examination by a rheumatologist) was not undertaken of all 48 of the non-recovered participants at 1 year. Thus, there could be fibromyalgia cases missed. The Modified 2010 ACR criteria questionnaire has a $90 \%$ sensitivity for a diagnosis of fibromyalgia performed by a rheumatologist as the gold standard. ${ }^{17}$ Thus, an additional four or five cases could have been missed out of 48 who failed to meet the Modified 2010 ACR criteria for fibromyalgia. Yet, looking at the questionnaire scores, which were relatively low, this seems unlikely. Another limitation is that not all participants were female, and one might suppose that women would 
be more likely to develop fibromyalgia, given the generally observed female preponderance. Examining only the incidence in female participants, however, there was 1 in 144 cases of fibromyalgia $(0.7 \%)$. Since some whiplash-injured participants could have widespread pain immediately after the collision, unless they had a precollision history or confirmed diagnosis of fibromyalgia or widespread pain, they were not excluded. It is true that this may have biased the study towards potential over-inclusion of pre-existing fibromyalgia if the initial postcollision presentation with widespread pain was not arising from the collision itself. Yet, this also prevented inappropriate exclusion of patients whose initial presentations may in fact have been a risk factor for fibromyalgia in the future (eg, high pain intensity and more symptoms is a risk factor for poor prognosis in whiplash injury). ${ }^{22}$ Finally, it is possible that the development of fibromyalgia requires more time than 1 year. However, it is difficult to postulate a mechanism that would delay by more than a year the onset of fibromyalgia after trauma and well after established, chronic pain.

Littlejohn and Guymer ${ }^{7}$ have suggested that chronic whiplash is the same elephant as fibromyalgia but in a different room, and that these patients should be all given a diagnosis of fibromyalgia. However, labelling a patient with isolated, chronic neck pain or isolated low back pain, even if disabling, with a diagnosis of fibromyalgia does not seem helpful. Though it is clearly possible to diagnose fibromyalgia without meeting any standard criteria, if every chronic pain patient is labelled with fibromyalgia, the diagnosis becomes relatively meaningless, and so too does the question of how often whiplash injury leads to fibromyalgia.

Contributors RF is responsible for design and study conception, data collection, data analysis, interpretation of data and writing of paper.

Competing interests None.

Ethics approval Alberta Health Research Ethics Board.

Provenance and peer review Not commissioned; externally peer reviewed.

Data sharing statement No additional data are available.

Open Access This is an Open Access article distributed in accordance with the Creative Commons Attribution Non Commercial (CC BY-NC 4.0) license, which permits others to distribute, remix, adapt, build upon this work noncommercially, and license their derivative works on different terms, provided the original work is properly cited and the use is non-commercial. See: http:// creativecommons.org/licenses/by-nc/4.0/

\section{REFERENCES}

1. Ferrari R. Predicting central sensitization-whiplash patients. Aust Fam Physician 2010;39:863-6.

2. Ferrari R, Louw D. Coping style as a predictor of compliance with referral to active rehabilitation in whiplash patients. Clin Rheumatol 2011;30:1221-5.

3. Ferrari R. Correlation between self-reported recovery and central sensitization in whiplash patients. $J$ Sport Health Sci 2012;1:61-4.

4. Ferrari R, Louw $D$. Effect of a pain diary use on recovery from acute whiplash injury, a cohort study. J Zhejiang Univ-Sc $B$ 2013;14:1049-53.

5. Ferrari R. Predicting recovery from whiplash injury in the primary care setting. Aust Fam Physician 2014;43:559-62.

6. Ferrari R. A prospective study of perceived injustice in whiplash victims and its relationship to recovery. Clin Rheumatol 2014. [Epub ahead of print 3 Jun 2014].

7. Littlejohn GO, Guymer EK. Whiplash: same elephant, different room. $J$ Rheumatol 2014;41:411-13.

8. Wolfe F, Häuser W, Walitt B, et al. Fibromyalgia and physical trauma: the concepts we invent. J Rheumatol 2014;41:1737-45.

9. Buskila D, Neumann L, Vaisberg G, et al. Increased rates of fibromyalgia following cervical spine injury. A controlled study of 161 cases of traumatic injury [see comments]. Arthritis Rheum 1997;40:446-52.

10. Tishler M, Levy O, Amit-Vazina M. Can fibromyalgia be associated with whiplash injury? A 3-year follow-up study. Rheumatol Int 2011;31:1209-13.

11. Virani S, Ferrari R, Russell AS. Physician resistance to the late whiplash syndrome. J Rheumatol 2001;28:2096-9.

12. Ferrari R, Russell AS. Whiplash: social interventions and solutions. J Rheumatol 2008;35:2300-2.

13. Spitzer WO, Skovron ML, Salmi LR, et al. Scientific monograph of the Quebec Task Force on Whiplash-Associated Disorders: redefining "whiplash" and its management. Spine 1995;20(8 Suppl):1S-73S.

14. Wolfe F, Smythe HA, Yunus MB, et al. The American College of Rheumatology 1990 Criteria for the Classification of Fibromyalgia. Report of the Multicenter Criteria Committee. Arthritis Rheum 1990;33:160-72.

15. Wolfe F, Clauw DJ, Fitzcharles MA, et al. Fibromyalgia criteria and severity scales for clinical and epidemiological studies: a modification of the ACR Preliminary Diagnostic Criteria for Fibromyalgia. $J$ Rheumatol 2011;38:1113-22.

16. Ferrari R, Russell AS, Kelly AJ. Assessing whiplash recovery-the Whiplash Disability Questionnaire. Aust Fam Physicain 2006;35:653-4.

17. Wolfe F, Brähler E, Hinz A, et al. Fibromyalgia prevalence, somaticsymptom reporting, and the dimensionality of polysymptomatic distress: results from a survey of the general population. Arthritis Care Res (Hoboken) 2013;65:777-85.

18. Ferrari R, Russell AS. A questionnaire using the modified 2010 American College of rheumatology criteria for fibromyalgia: specificity and sensitivity in clinical practice. $J$ Rheumatol 2013;40:1590-5.

19. Croft P, Rigby AS, Boswell R, et al. The prevalence of chronic widespread pain in the general population. $J$ Rheumatol 1993;20:710-13.

20. Croft P, Schollum J, Silman A. Population study of tender point counts and pain as evidence of fibromyalgia. BMJ 1994:309:696-9.

21. Wiltshire J. Samuel Johnson in the medical world. The doctor and the patient. New York: Cambridge University Press, 1991:113.

22. Carroll LJ, Holm L, Ferrari R, et al. Recovery in whiplash-associated disorders: do you get what you expect? J Rheumatol 2009;36:1063-70. 